\title{
Melt-Processable Nanocomposites Grafting-From Platelet Surfaces by Vapor-Assisted Surface Polymerization
}

\author{
Yoshito Andou and Haruo Nishida \\ Eco-Town Collaborative R\&D Center for the Environment and Recycling, Kyushu Institute of Technology, 2-4 Hibikino, \\ Wakamatsu-ku, Kitakyushu, Fukuoka 808-0196, Japan \\ Correspondence should be addressed to Haruo Nishida, nishida@lsse.kyutech.ac.jp
}

Received 14 July 2011; Accepted 2 August 2011

Academic Editors: E. Le Bourhis, B. Tan, and A. Taubert

Copyright (๑) 2011 Y. Andou and H. Nishida. This is an open access article distributed under the Creative Commons Attribution License, which permits unrestricted use, distribution, and reproduction in any medium, provided the original work is properly cited.

\begin{abstract}
One issue accompanying the melt-processing polymer/clay nanocomposites is the reaggregation of silicate platelets, which induces decreases in advantages of nanocomposites. To address this issue, vapor-assisted surface polymerization (VASP) method was applied with an initiator-attached and copolymerizable surfactant moiety-bound A-C18/C6MMT to obtain the exfoliated and intercalated nanocomposites using methylmethacrylate and styrene as vinyl monomers, respectively. The melt processing of the nanocomposites was carried out by a melt-compression molding method at $200^{\circ} \mathrm{C}$. From XRD measurements, the C18/C6MMTbased nanocomposites showed no change in $d$-spacing even after melt processing, indicating the maintenance of the exfoliation and intercalation states. This maintenance must result from polymer chains grafting from the silicate layer surfaces, thus clearly confirming the anchoring effect of the copolymerizable surfactant moiety units.
\end{abstract}

\section{Introduction}

Polymer/clay nanocomposites have brought about significant improvements in optical [1], mechanical [2,3], flameresistant [4-7], and gas barrier [8-10] properties over their corresponding pristine polymers and as such have been extensively studied. Moreover, because the improvements are expressed even at low concentrations of clay, the transparency of the original polymers is preserved. The property improvements are strongly dependent on the intercalation and exfoliation of the silicate platelets of clay. These layered silicates, because of heterovalent substitutions residing in octahedral layers [11], have a negative charge and this negative charge extends throughout the whole lattice, forcing counter cations to distribute within the interior of galleries. Counter cations, such as $\mathrm{Na}^{+}, \mathrm{Li}^{+}$, and $\mathrm{Ca}^{2+}$ reside in interlayer spaces at a basal $d$-spacing with the spacing distance determined by the cation's nature.

The most common way for facilitating the exfoliation or intercalation of silicate layers is by exchanging the small alkali and alkali earth cations in the gallery spaces for organic ammonium and imidazolium salts having long alkyl chains, for example, octadecylammonium chloride $[12,13]$ and 1,2-dimethy-3-hexadecylimidazolium salts [14]. The introduction of organic salts changes the chemical properties of silicate layer surfaces and increases their compatibility with polymers. The silicate layer surfaces of montmorillonite (MMT) as typical clay have been modified by various controlled living polymerization techniques, such as atom transfer radical polymerization [15-18], reversible additionfragmentation chain transfer polymerization [19-21], and nitroxide-mediated polymerization [22-24]. These polymerizations take place in the galleries with growing polymer chains spreading the distance between the silicate layers.

To produce plastic moldings, various melt-processing methods such as extrusion and injection molding are generally used. Exfoliated silicate platelets easily aggregate to return to layered structures after melt processing. It is important, therefore, to maintain the absolutely exfoliated state of silicate platelets even after melt processing is completed. Approaches for preserving the exfoliated state are expected to involve the grafting method of polymer chains from or onto silicate platelet surfaces, requiring at least one of 
the chain ends or internal chain units to be anchored to the silicate layer surfaces. Weimer et al. [22] demonstrated that the in situ alkoxyamine or nitroxide-mediated living radical polymerization from silicate-anchored initiators was effective in producing exfoliated polystyrene/layered silicate nanocomposites. This exfoliated structure was preserved even after the subsequent melt processing.

The vapor-phase-assisted surface polymerization (VASP) technique has been developed as a simple precise and solventless method for covering solid substrate surfaces by polymer layers [25]. One attractive feature of the VASP method is that gaseous monomers can diffuse and penetrate interstitially within the fine gaps and spaces of the solid substrates, allowing the construction of fine structured composites [26] and coatings [27, 28]. After the diffusion and adsorption on the interstitial surfaces, the monomers polymerize in a manner of "pseudografting from" the substrate surfaces. Polymer chains then grow on the surfaces by filling the spaces. Recently, a simple method for construction of vinyl polymer/clay nanocomposites by VASP of methyl methacrylate (MMA) was reported, by which a completely exfoliated poly(methyl methacrylate) (PMMA)/MMT nanocomposite was obtained [29]. It was also revealed that, because of a particular interaction between silicate platelet surfaces and MMA units in copolymer chains, a small number of MMA units in a styrene-MMA copolymer induced the exfoliation of layered silicates [30]. Considering the easy preparation of block copolymers and other advantageous features of VASP method [31], various designs of polymer structure by VASP could be created on the silicate layer surfaces to prepare nanocomposites that maintain their structures after melt processing.

In this report, the grafting of polymer chains from the silicate layer surfaces of MMT was studied by VASP to prepare the nanocomposites, with changes in the nanostructures of composites also investigated. The silicate layer surfaces were modified with a copolymerizable organic cation: 2-methacryloyloxyhexyltriethylammonium bromide and followed by VASP of vinyl monomers. Nanostructures and their changes after melt processing were analyzed to clarify effects of the VASP method on the exfoliation of silicate platelets and their subsequent preservation.

\section{Experimental}

2.1. Materials. Monomer, methyl methacrylate (MMA, 99.0\%, from Tokyo Chemical Industry Co., Ltd.), and styrene (St, 99\%, from Wako Pure Chemical Industries, Ltd., Wako) were purified by distillation under reduced pressure over $\mathrm{CaH}_{2}$ just before polymerization. Initiator, $2,2^{\prime}$-azobis(isobutyronitrile) (AIBN, $>99 \%$ ) was purchased from Otsuka Chemical Inc., and crystallized from methanol. Polymerization inhibitor, 4-tert-butylpyrocatechol (>98\%), was purchased from Wako, and used as received. Substrate, sodium montmorillonite (Kunipia F) was used as received from Kunimine Industries Co., Ltd. Poly(methyl methacrylate) (PMMA, $\left.M_{n} 5.0 \times 10^{4}, M_{w} 7.0 \times 10^{4}\right)$ was purchased from Wako and used as received. Poly(styrene) (PSt, $M_{w} 2.8 \times$ $10^{5}$ ) was purchased from Aldrich. All other reagents, such as acetone (>99\%), chloroform $\left(\mathrm{CHCl}_{3},>99.0 \%\right.$, HPLC grade), and methanol (>99\%) were commercially obtained and purified by distillation.

\subsection{Preparation of 2-Methacryloyloxyhexyltriethylammonium} Bromide (MHAB). In a round-bottom flask, 6-bromo-1hexanol $4.0 \mathrm{~g}(21.43 \mathrm{mmol})$ methacryloylchloride $3.5 \mathrm{~mL}$ (32.15 mmol), $\mathrm{CHCl}_{3} 10 \mathrm{~mL}$ were added and stirred at $25^{\circ} \mathrm{C}$ for $24 \mathrm{~h}$. After the reaction, the mixture was washed with $10 \mathrm{~mL}$ of water three times and the solvent evaporated. Triethyl amine $6.0 \mathrm{~mL}(54.3 \mathrm{mmol})$ was dropped into the residual viscous product and the mixture was stirred at $25^{\circ} \mathrm{C}$ for $10 \mathrm{~min}$. Acetone $(10 \mathrm{~mL})$ was added to the mixture, and then 2-methacryloyloxyhexyltriethylammonium bromide (MHAB) was precipitated as a white powder. The powdery crude product was filtered, washed with acetone several times, and dried overnight in vacuo, resulting in obtaining $0.30 \mathrm{~g}$ of pure product:

${ }^{1} \mathrm{H}$ NMR $\left(\mathrm{CD}_{3} \mathrm{OD}\right): \delta 6.07(\mathrm{~d}, 1 \mathrm{H}), 5.60(\mathrm{~d}, 1 \mathrm{H}), 4.14(\mathrm{t}$, $2 \mathrm{H}), 3.45(\mathrm{t}, 2 \mathrm{H}), 3.20(\mathrm{q}, 6 \mathrm{H}), 1.98(\mathrm{~s}, 3 \mathrm{H}), 1.86(\mathrm{~m}, 2 \mathrm{H})$, $1.72(\mathrm{~m}, 2 \mathrm{H}), 1.50(\mathrm{br}, 4 \mathrm{H}), 1.45(\mathrm{t}, 9 \mathrm{H})$.

\subsection{Preparation of Organically Modified Montmorillonite.} Typical procedure. Sodium MMT (Kunipia F, $2.0 \mathrm{~g}, d-$ spacing calculated from (001) basal plane diffraction: 1.20 $\mathrm{nm})$ was dispersed in distilled water $(2 \mathrm{~L})$ and stirred overnight at room temperature. Triethylstearyl ammonium chloride $(\mathrm{C} 18,2.15 \mathrm{mmol})$ and 2-methacryloyloxyhexyltriethyl ammonium bromide (MHAB (C6), $0.57 \mathrm{mmol}$ ) were dissolved in $200 \mathrm{~mL}$ of distilled water, and the solution slowly added into the MMT suspension solution. After stirring for $3 \mathrm{~h}$ at room temperature, the suspension was filtered to isolate precipitates. The precipitates were washed with distilled water until no halide ions could be detected by an aqueous $\mathrm{AgNO}_{3}$ solution, dried overnight at $50^{\circ} \mathrm{C}$ in $v a c u o$, and crushed into powders in a mortar, resulting in the preparation of organically modified MMT (C18/C6MMT, $2.41 \mathrm{~g}$ ). The same procedure was used to prepare C18MMT.

\subsection{Preparation of Initiator-Intercalated Organically Modi-} fied MMT. Typical procedure: initiator AIBN intercalated C18/C6MMT (A-C18/C6MMT) was prepared in a similar manner as reported elsewhere in detail [29]. Before VASP, to intercalate the initiator AIBN into the silicate layers, C18/ C6MMT $(2.41 \mathrm{~g})$ was treated with a $1 \mathrm{mM}$ acetone solution $(500 \mathrm{~mL})$ of AIBN at a $1: 30$ weight ratio to C18/C6MMT at $25^{\circ} \mathrm{C}$ for $0.5 \mathrm{~h}$ under stirring. After the treatment, acetone was removed under vacuum at room temperature, resulting in the production of powdery A-C18/C6MMT. The crude AC18/C6MMT powder was stirred in $\mathrm{CHCl}_{3}$ to wash out the initiators attached to outside surfaces. The same procedure was used to prepare AIBN-intercalated A-C18MMT.

2.5. Typical Procedure of VASP. VASP of MMA was carried out in an $\mathrm{H}$-shaped glass tube reactor with a vacuum cock. AC18/C6MMT (100 mg) was added into a glass pan (bottom surface area: $154 \mathrm{~mm}^{2}$ ), and the glass pan set in the bottom of one of the legs of the $\mathrm{H}$-shaped glass tube reactor. 


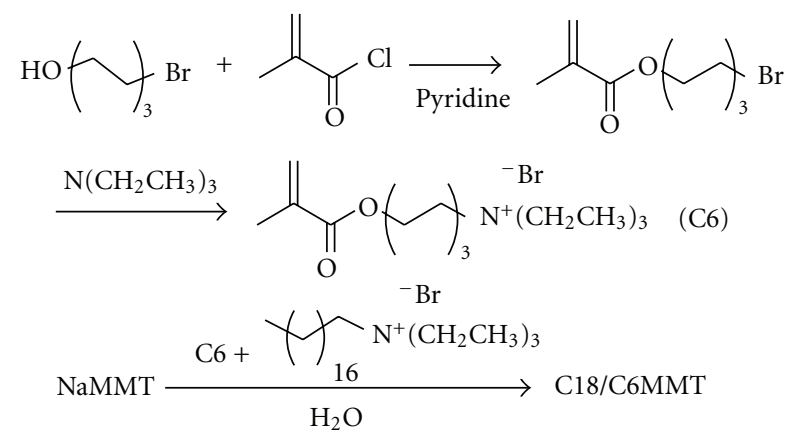

Scheme 1: Synthesis of modified MMT: C18/C6MMT.
MMA (2.0 mL) and 4-tert-butylpyrocatechol $(20 \mathrm{mg}, 1.2 \times$ $10^{-4} \mathrm{~mol}$ ) were introduced into the bottom of the other leg. The reactor was degassed by three freeze-pump-thaw cycles and then sealed under a saturated atmosphere of vaporized MMA. Polymerization was carried out at $70^{\circ} \mathrm{C}$ for $3 \mathrm{~h}$ under a saturated vapor pressure of $3.27 \times 10^{4} \mathrm{~Pa}$ in a thermostated oven. After the reaction, the sample, which was expanded by newly intercalated polymer chains, was dried to remove the adsorbed MMA in vacuo and weighed to obtain $613 \mathrm{mg}$ of PMMA-C18/C6MMT composite. The produced composite was analyzed intact with XRD to measure $d$-spacing values of silicate layers.

2.6. Melt Processing. Melt processing of composites was carried out by a compression molding method, in which mixtures of composite powders and homopolymers were preheated for $2 \mathrm{~min}$ in an oil press heated at $200^{\circ} \mathrm{C}$, followed by heat-kneading for $3 \mathrm{~min}$, and heat-pressing to obtain a thin film (thickness ca. $100 \mu \mathrm{m}$ ).

2.7. Characterization Methods. X-ray diffraction (XRD) patterns were obtained by using a Rigaku diffractometer equipped with a $\mathrm{Cu} \mathrm{K} \alpha$ generator $(\lambda 0.1541 \mathrm{~nm})$ under the following conditions: slit width, $0.30 \mathrm{~mm}$; generator current, $16 \mathrm{~mA}$; voltage, $30 \mathrm{kV}$; scanning rate, 2 degree $\cdot \mathrm{min}^{-1}$.

\section{Results and Discussion}

3.1. Preparation of Initiator-Intercalated Organically Modified MMT. To easily achieve radical polymerization at the intergalleries and to anchor polymer chains on silicate layer surfaces, triethylstearyl ammonium (C18) and polymerizable methacryloyloxyhexylammonium (C6) moieties were immobilized by exchanging with alkali and alkali earth counter cations on the surfaces. Preparation pathways are shown in Scheme 1. Previously, it was confirmed that an acetone solution of AIBN smoothly diffused into C18modified silicate interlayers, and that the initiator remained in the interlayers after drying the silicates [29]. Insertion of AIBN into the silicate interlayers successfully proceeded, with the expected increase in interlayer $d$-spacing. After the surface-modification and the AIBN insertion, XRD profile of the treated MMTs shifted to a lower $2 \theta$ range

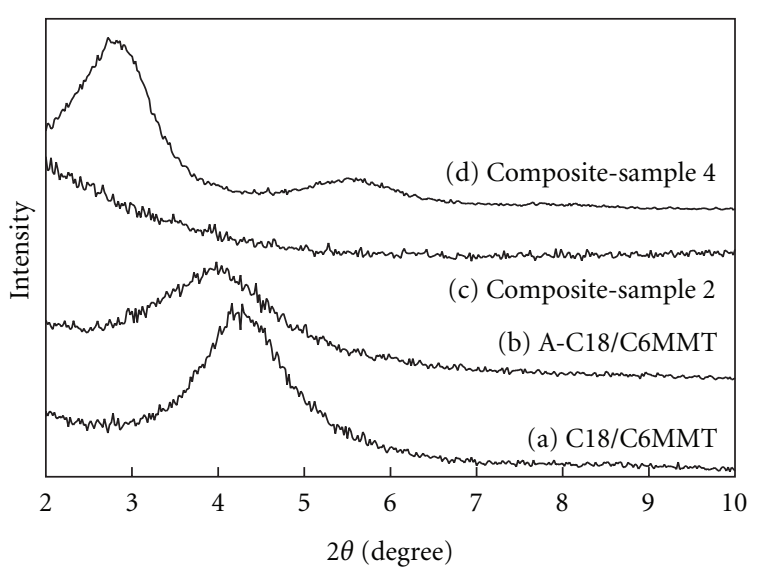

FIGURE 1: XRD profiles of composites by VASP of MMA and St with modified C18/C6MMT. (a) C18/C6MMT, (b) A-C18/C6MMT, (c) composite-sample 2, and (d) composite-sample 4.

than that of original MMT as shown in Figure 1, resulting in further increases in the $d$-spacing between the silicate layers from 1.20 to $2.05 \mathrm{~nm}$ after the C18/C6-modification (C18/C6MMT) and to $2.18 \mathrm{~nm}$ after the AIBN insertion (A-C18/C6MMT). In the case of C18MMT, the $d$-spacing increased to $2.20 \mathrm{~nm}$ after the C18-modification (C18MMT) and to $2.30 \mathrm{~nm}$ after the AIBN insertion (A-C18MMT) [30].

3.2. VASP of Vinyl Monomers with Initiator-Intercalated Organically Modified MMT. VASP of vinyl monomers: MMA and St was performed with A-C18MMT and A-C18/C6MMT (modified MMT: AIBN = 30: $1 \mathrm{wt} / \mathrm{wt}$ ). After VASP, obtained composites were analyzed by an X-ray diffractometer (XRD) to evaluate the exfoliation or intercalation of layered silicates. Here, the exfoliation was defined as resulting in a $d$-spacing of greater than $4.4 \mathrm{~nm}$ at the limit of the $2 \theta$ value $\left(2.0^{\circ}\right)$ measurable with the XRD. Conditions and results of VASP are listed in Table 1. As references, VASP of vinyl monomers with C18/C6MMT were also carried out.

During VASP, AIBN-intercalated MMTs gradually expanded with time, suggesting that polymerization took place around the silicate layers. For example, VASP of MMA on A-C18/C6MMT (sample 2) showed a high increment of $498.3 \mathrm{wt} \%$, which meant that the product comprised $83.3 \mathrm{wt}$ $\%$ of newly generated material and $16.7 \mathrm{wt} \%$ of the original C18/C6MMT. No change in weight was observed on $[1,2]$ in Table 1 using C18/C6MMT without radical initiator. From XRD measurements, VASP samples of MMA on A-C18MMT and A-C18/C6MMT (samples 1 and 2) were confirmed to be exfoliated structures because of no $2 \theta$ peak being apparent in XRD profiles (Figure 1). This means that polymerization occurs at silicate interlayer spaces and that growing polymer chains spread out forcing the silicate layers to move apart from each other.

VASP of St on A-C18MMT and A-C18/C6MMT (samples 3 and 4) proceeded slowly with gradual expansion in volume of the modified MMT powders. Although XRD profiles of obtained VASP samples showed drastic shifts into lower $2 \theta$ 
TABLE 1: VASP of methyl methacrylate and styrene on modified MMTs.

\begin{tabular}{|c|c|c|c|c|c|c|}
\hline Sample & Modified MMT & Monomer & Time/h & Temp. $/{ }^{\circ} \mathrm{C}$ & Increment/wt $\%^{\mathrm{a}}$ & $d$-spacing/nm \\
\hline 1 & A-C18MMT & MMA & 3 & 70 & 564.9 & Exfoliation \\
\hline 2 & A-C18/C6MMT & MMA & 3 & 70 & 498.3 & Exfoliation \\
\hline$[1]$ & C18/C6MMT & MMA & 3 & 70 & 0.0 & - \\
\hline 3 & A-C18MMT & St & 20 & 70 & 127.7 & 3.90 \\
\hline 4 & A-C18/C6MMT & St & 20 & 70 & 182.0 & 3.17 \\
\hline$[2]$ & C18/C6MMT & St & 20 & 70 & 0.0 & - \\
\hline
\end{tabular}

${ }^{\mathrm{a}}$ Increment of accumulated polymer to modified MMT.

TABLE 2: Melt processing of blends of VASP samples and homopolymers.

\begin{tabular}{|c|c|c|c|c|}
\hline Sample & Composite/g & Homopolymer ${ }^{\mathrm{a}} \mathrm{g}$ & $\mathrm{MMT} / \mathrm{wt} \%$ & $d$-spacing/nm \\
\hline 5 & Sample $1 / 0.11$ & PMMA/0.17 & 5.8 & 3.34 \\
\hline 6 & Sample $2 / 0.10$ & PMMA/0.18 & 6.0 & 4.12 \\
\hline 7 & Sample $3 / 0.11$ & PSt/0.50 & 7.8 & 3.11 \\
\hline 8 & Sample 4/0.05 & PSt/0.32 & 4.8 & 3.17 \\
\hline
\end{tabular}

${ }^{a}$ Homopolymer: PMMA $M_{n} 5.0 \times 10^{4}, M_{w} 7.0 \times 10^{4}$; PSt $M_{w} 2.8 \times 10^{5}$.

ranges than those of the modified MMTs (Figure 1), the increases in the $d$-spacing values to 3.90 (sample 3 ) and $3.17 \mathrm{~nm}$ (sample 4) from 2.30 (A-C18MMT) and $2.18 \mathrm{~nm}$ (A-C18/C6MMT) indicate intercalation of the structure, rather than exfoliation. As previously reported [30], the exfoliation/intercalation is affected by the compatibility between monomeric units in copolymers and the silicate interlayer components. In this case, such compatibility may be the primary factor for the exfoliation rather than the expected anchoring effect of the C6 moiety bound on the silicate layer surfaces.

3.3. Anchoring Effect of Methacryloyloxyhexylammonium (C6) Moiety on Melt Processing. Melt processing of plastics is an indispensable process for producing various moldings. It is known that exfoliated silicate platelets reaggregate to return to layered structures after melt processing has completed [32]. To inhibit the return to the layered structure, copolymerizable methacryloyloxyhexylammonium (C6) moieties were introduced on the silicate layer surfaces. The effects of the C6 moiety were verified by melt-blending composite samples in Table 1 and homopolymers so as to give similar quantities of MMT (4.8-7.8 wt\%; Table 2). The blend samples were prepared by a compression molding method at $200^{\circ} \mathrm{C}$, and obtained melt-blend samples were analyzed by $\mathrm{XRD}$ to evaluate the changes in $d$-spacing values. XRD profiles are shown in Figures 2 and 3.

Figure 2 shows changes in the XRD profiles of the C18MMT-based composite blend samples: (a) compositesample 1/PMMA (sample 5) and (b) composite-sample 3/PSt (sample 7), compared with the original composites (samples 1 and 3). As listed in Table 2, both melt-blend samples clearly showed decreases in the $d$-spacing values from exfoliation state to $3.34 \mathrm{~nm}$ (sample 5) and from $3.90 \mathrm{~nm}$ to $3.11 \mathrm{~nm}$ (sample 7), indicating the aggregation of silicate platelets and a compression of the layered structure, respectively. However, the layered structure of C18MMT was not recovered until the original value ( $d$-spacing $2.30 \mathrm{~nm}$ ) was reached. As reported previously [29], a few polymer chains may be covalently bound on the silicate surfaces and the weak anchoring effect of these grafted chains may explain incomplete recoveries of $d$-spacing values after the melt processing.

Figure 3 shows changes in XRD profiles of blended C18/ C6MMT-based composite samples: (a) composite-sample 2/PMMA (sample 6) and (b) composite-sample 4/PSt (sample 8), in comparison with the original composite samples (samples 2 and 4). Interestingly, it was found that the XRD peaks of blended C18/C6MMT-based composite samples were hardly changed. This maintenance of the $d$-spacing after melt processing must result from the typical anchoring effect of the C6 moieties bound on the silicate layer surfaces. During VASP of vinyl monomers with A-C18/C6MMT, gaseous vinyl monomers can diffuse into interlayer spaces of silicate layers and polymerize by radicals generated from AIBN causing expansion of the interlayer spaces. At the same time, the C6 moieties must also join the radical polymerization to copolymerize, resulting in the formation of anchors on the silicate surfaces.

In XRD profiles of the melt-processed C18/C6MMTbased composite samples (samples 6 and 8 ), small diffraction peaks newly appeared or enlarged at $2 \theta=5^{\circ}$ (sample 6) and $5.5^{\circ}$ (sample 8), respectively. This phenomenon suggests the aggregation of a small amount of silicate platelets after meltprocessing.

These results indicate that the copolymerization of C6 moieties proceeded considerably with the anchoring effect on silicate layer surfaces and the resulting inhibition of the reaggregation of silicate platelets clearly demonstrated. The anchoring effect was confirmed by using both monomers: MMA and St in the same manner. Thus, these anchoring 


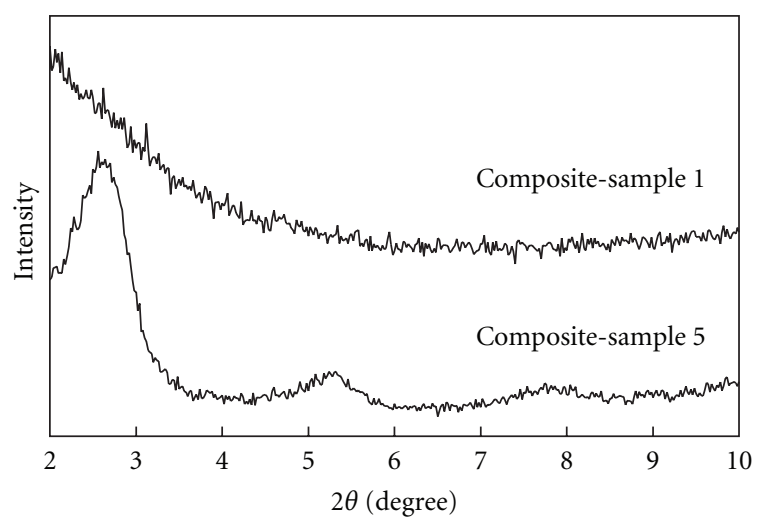

(a)

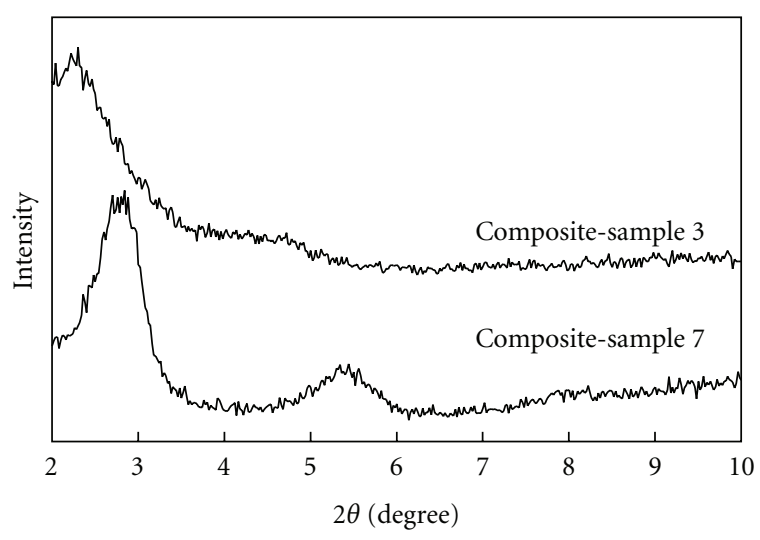

(b)

Figure 2: Changes in XRD peaks of C18MMT-based composite/homopolymer blends before and after melt processing. (a) composite-sample 1/PMMA, (b) composite-sample 3/PSt.

effects must be common to vinyl monomers copolymerizable with C6 moiety.

\section{Conclusions}

To address the reaggregation of silicate platelets after the melt processing, C18/C6MMT, modified with a long alkyl chain surfactant moiety: triethylstearyl ammonium $(\mathrm{C} 18)$ and a copolymerizable surfactant moiety: 2-methacryloyloxyhexyltriethyl ammonium (C6), was synthesized. VASP of MMA and St was carried out with the AIBN-supporting AC18/C6MMT and successfully proceeded to obtain exfoliated and intercalated nanocomposites, respectively. The obtained nanocomposites were melt blended with corresponding homopolymers by the melt-compression molding method. XRD peaks of the C18/C6MMT-based nanocomposites never shifted into a high $2 \theta$ range even after the melt processing. On the other hand, when another C18MMT without C6 moiety was used, similar exfoliated and intercalated nanocomposites were obtained by VASP of MMA and St, respectively. However, considerable changes in XRD peaks of the C18MMT-based nanocomposites were confirmed after the melt processing, shifting into high $2 \theta$ ranges. These

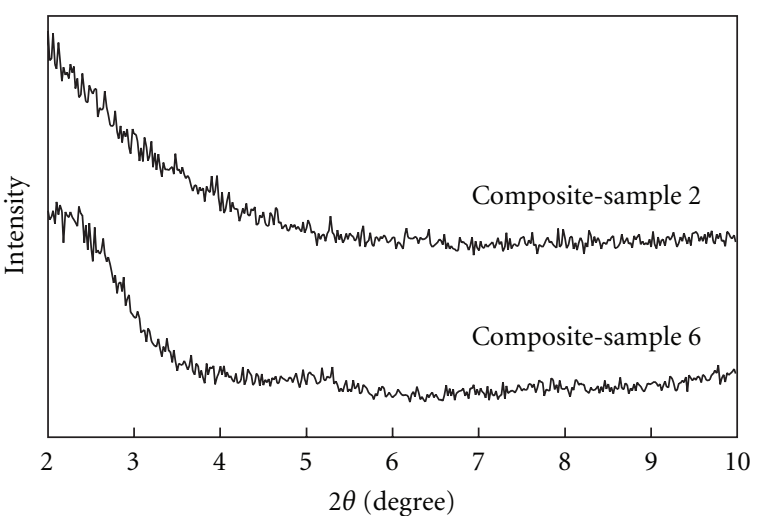

(a)

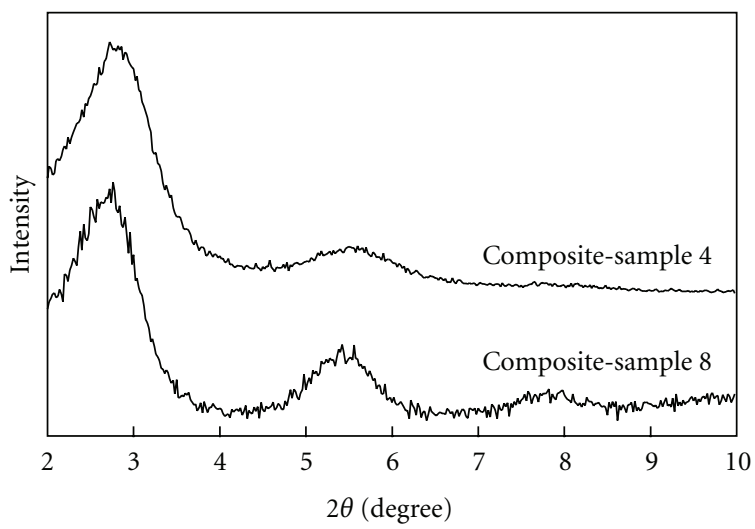

(b)

FIgure 3: Changes in XRD peaks of C18/C6MMT-based composite/homopolymer blends before and after melt processing. (a) composite-sample 2/PMMA, (b) composite-sample 4/PSt.

results clearly demonstrate the anchoring effect of C6 moiety to the silicate layer surfaces.

\section{References}

[1] L. Cui, N. H. Tarte, and S. I. Woo, "Effects of modified clay on the morphology and properties of PMMA/clay nanocomposites synthesized by in situ polymerization," Macromolecules, vol. 41, no. 12, pp. 4268-4274, 2008.

[2] F. Chavarria and D. R. Paul, "Morphology and properties of thermoplastic polyurethane nanocomposites: effect of organoclay structure," Polymer, vol. 47, no. 22, pp. 7760-7773, 2006.

[3] Z. Wang and T. J. Pinnavaia, "Nanolayer reinforcement of elastomeric polyurethane," Chemistry of Materials, vol. 10, no. 12, pp. 3769-3771, 1998.

[4] G. Beyer, "Nanocomposites: a new class of flame retardants for polymers," Plastics, Additives and Compounding, vol. 4, no. 10, pp. 22-28, 2002.

[5] X. Zheng and C. A. Wilkie, "Flame retardancy of polystyrene nanocomposites based on an oligomeric organically-modified clay containing phosphate," Polymer Degradation and Stability, vol. 81, no. 3, pp. 539-550, 2003.

[6] X. Zheng, D. D. Jiang, and C. A. Wilkie, "Methyl methacrylate oligomerically-modified clay and its poly(methyl meth- 
acrylate) nanocomposites," Thermochimica Acta, vol. 435, no. 2, pp. 202-208, 2005.

[7] M. Si, M. Goldman, G. Rudomen, M. Y. Geifer, J. C. Sokolov, and M. H. Rafailovich, "Effect of clay type on structure and properties of poly(methyl methacrylate)/clay nanocomposites," Macromolecular Materials and Engineering, vol. 291, no. 6, pp. 602-611, 2006.

[8] H. K. Jeong, W. Krych, H. Ramanan, S. Nair, E. Marand, and M. Tsapatsis, "Fabrication of polymer/selective-flake nanocomposite membranes and their use in gas separation," Chemistry of Materials, vol. 16, no. 20, pp. 3838-3845, 2004.

[9] T. Ogasawara, Y. Ishida, T. Ishikawa, T. Aoki, and T. Ogura, "Helium gas permeability of montmorillonite/epoxy nanocomposites," Composites Part A, vol. 37, no. 12, pp. 22362240, 2006.

[10] B. Xu, Q. Zheng, Y. Song, and Y. Shangguan, "Calculating barrier properties of polymer/clay nanocomposites: effects of clay layers," Polymer, vol. 47, no. 8, pp. 2904-2910, 2006.

[11] P. V. Brady and J. L. Krumhansl, "The surface chemistry of clay minerals," in Oxide Surface, Surfactant Science Series 103, J. A. Wingrave, Ed., pp. 281-302, Marcel Dekker, New York, NY, USA, 2001.

[12] R. A. Vaia, R. K. Teukolsky, and E. P. Giannelis, "Interlayer structure and molecular environment of alkylammonium layered silicates," Chemistry of Materials, vol. 6, no. 7, pp. 1017-1022, 1994.

[13] J. W. Gilman, C. L. Jackson, A. B. Morgan et al., "Flammability properties of polymer-layered-silicate nanocomposites. Polypropylene and polystyrene nanocomposites," Chemistry of Materials, vol. 12, no. 7, pp. 1866-1873, 2000.

[14] J. W. Gilman, W. H. Awad, R. D. Davis et al., "Polymer/ layered silicate nanocomposites from thermally stable trialkylimidazolium-treated montmorillonite," Chemistry of Materials, vol. 14, no. 9, pp. 3776-3785, 2002.

[15] H. Böttcher, M. L. Hallensleben, S. Nuß, H. Wurm, J. Bauer, and P. Behrens, "Organic/inorganic hybrids by "living"/controlled ATRP grafting from layered silicates," Journal of Materials Chemistry, vol. 12, no. 5, pp. 1351-1354, 2002.

[16] H. Zhao and D. A. Shipp, "Preparation of poly(styrene-blockbutyl acrylate) block copolymer-silicate nanocomposites," Chemistry of Materials, vol. 15, no. 14, pp. 2693-2695, 2003.

[17] H. Zhao, B. P. Farrell, and D. A. Shipp, "Nanopatterns of poly(styrene-block-butyl acrylate) block copolymer brushes on the surfaces of exfoliated and intercalated clay layers," Polymer, vol. 45, no. 13, pp. 4473-4481, 2004.

[18] H. Zhao, S. D. Argoti, B. P. Farrell, and D. A. Shipp, "Polymersilicate nanocomposites produced by in situ atom transfer radical polymerization," Journal of Polymer Science, Part A, vol. 42, no. 4, pp. 916-924, 2004.

[19] N. Salem and D. A. Shipp, "Polymer-layered silicate nanocomposites prepared through in situ reversible additionfragmentation chain transfer (RAFT) polymerization," Polymer, vol. 46, no. 19, pp. 8573-8581, 2005.

[20] B. Q. Zhang, C. Y. Pan, C. Y. Hong, B. Luan, and P. J. Shi, "Reversible addition-fragmentation transfer polymerization in the presence of MMT immobilized amphoteric RAFT agent," Macromolecular Rapid Communications, vol. 27, no. 2, pp. 97-102, 2006.

[21] A. Samakande, R. D. Sanderson, and P. C. Hartmann, "Encapsulated clay particles in polystyrene by RAFT mediated miniemulsion polymerization," Journal of Polymer Science, Part A, vol. 46, no. 21, pp. 7114-7126, 2008.

[22] M. W. Weimer, H. Chen, E. P. Giannelis, and D. Y. Sogah, "Direct synthesis of dispersed nanocomposites by in situ living free radical polymerization using a silicate-anchored initiator," Journal of the American Chemical Society, vol. 121, no. 7, pp. 1615-1616, 1999.

[23] J. Di and D. Y. Sogah, "Exfoliated block copolymer/silicate nanocomposites by one-pot, one-step in-situ living polymerization from silicate-anchored multifunctional initiator," Macromolecules, vol. 39, no. 15, pp. 5052-5057, 2006.

[24] C. Konn, F. Morel, E. Beyou, P. Chaumont, and E. BourgeatLami, "Nitroxide-mediated polymerization of styrene initiated from the surface of laponite clay platelets," Macromolecules, vol. 40, no. 21, pp. 7464-7472, 2007.

[25] Y. Andou, J.-M. Jeong, M. Kaneko, H. Nishida, and T. Endo, "Hydrophobic cellulose fiber surfaces modified with 2,2,3,3,3- pentafluoropropylmethacrylate (FMA) by vaporphase-assisted photopolymerization," Polymer Journal, vol. 42, no. 6, pp. 519-524, 2010.

[26] H. Nishida, M. Yamashita, Y. Andou, J.-M. Jeong, and T. Endo, "Gas-phase assisted surface polymerization behavior of $\beta$-propiolactone on inorganic and organic substrates and consequent composite production," Macromolecular Materials and Engineering, vol. 290, no. 8, pp. 848-856, 2005.

[27] K. K. S. Lau, J. Bico, K. B. K. Teo et al., "Superhydrophobic carbon nanotube forests," Nano Letters, vol. 3, no. 12, pp. 1701-1705, 2003.

[28] M. Gupta, V. Kapur, N. M. Pinkerton, and K. K. Gleason, "Initiated Chemical Vapor Deposition (iCVD) of conformal polymeric nanocoatings for the surface modification of highaspect-ratio pores," Chemistry of Materials, vol. 20, no. 4, pp. 1646-1651, 2008.

[29] Y. Andou, J.-M. Jeong, S. Hiki, H. Nishida, and T. Endo, "Design of nanocomposites by vapor-phase assisted surface polymerization," Macromolecules, vol. 42, no. 3, pp. 768-772, 2009.

[30] Y. Andou, J.-M. Jeong, H. Nishida, and T. Endo, "Simple procedure for polystyrene-based nanocomposite preparation by vapor-phase-assisted surface polymerization," Macromolecules, vol. 42, no. 20, pp. 7930-7935, 2009.

[31] M. Yasutake, S. Hiki, Y. Andou, H. Nishida, and T. Endo, "Physically controlled radical polymerization of vaporized vinyl monomers on surfaces. Synthesis of block copolymers of methyl methacrylate and styrene with a conventional free radical initiator," Macromolecules, vol. 36, no. 16, pp. 59745981, 2003.

[32] X. Huang and W. J. Brittain, "Synthesis and characterization of PMMA nanocomposites by suspension and emulsion polymerization," Macromolecules, vol. 34, no. 10, pp. 32553260, 2001. 

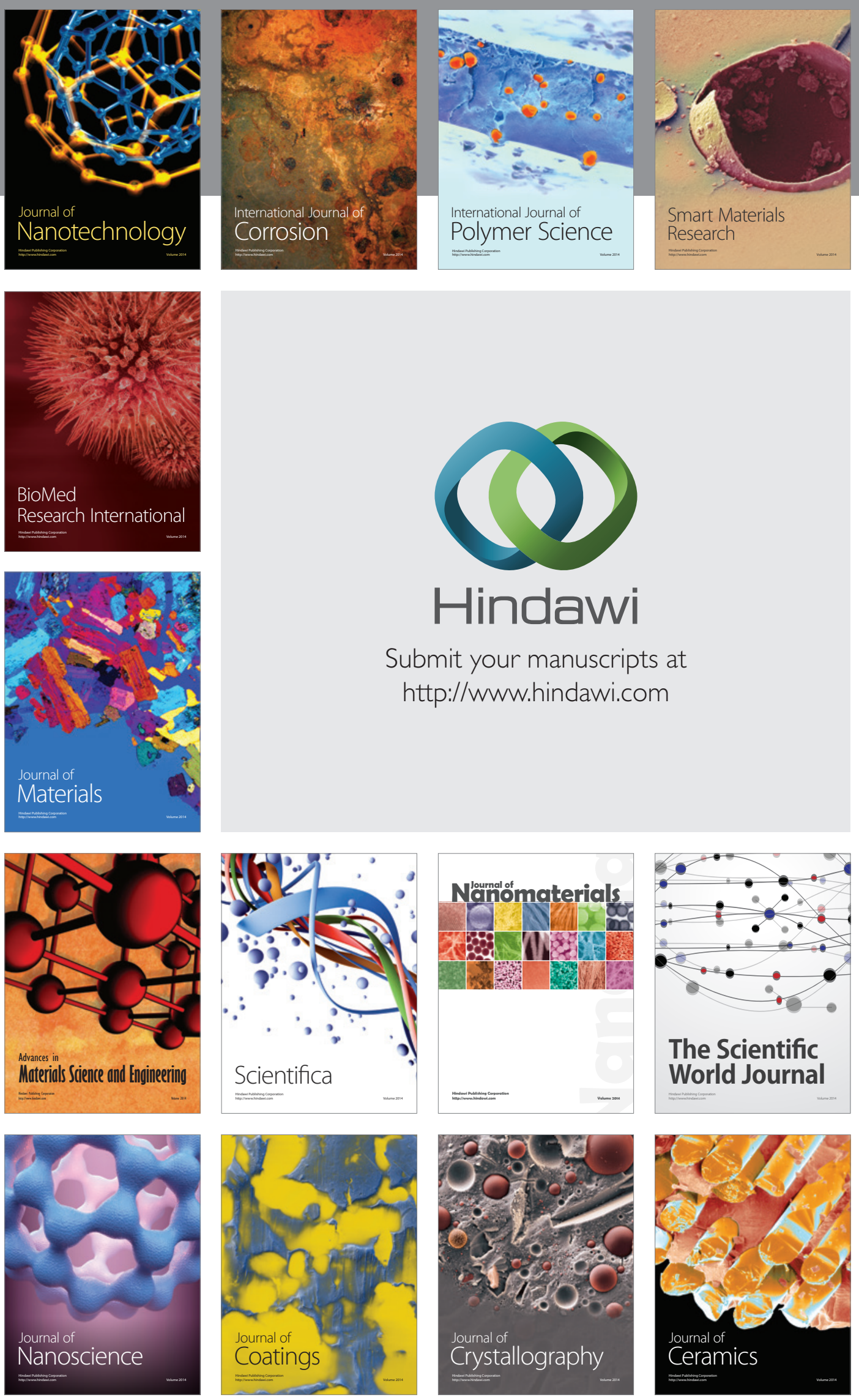

The Scientific World Journal

Submit your manuscripts at

http://www.hindawi.com

\section{World Journal}

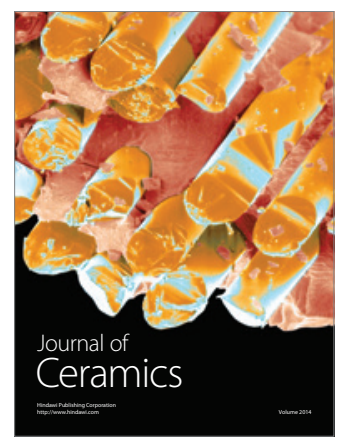

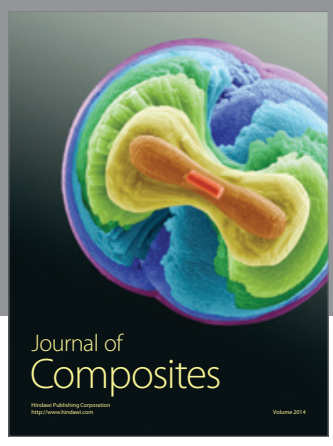
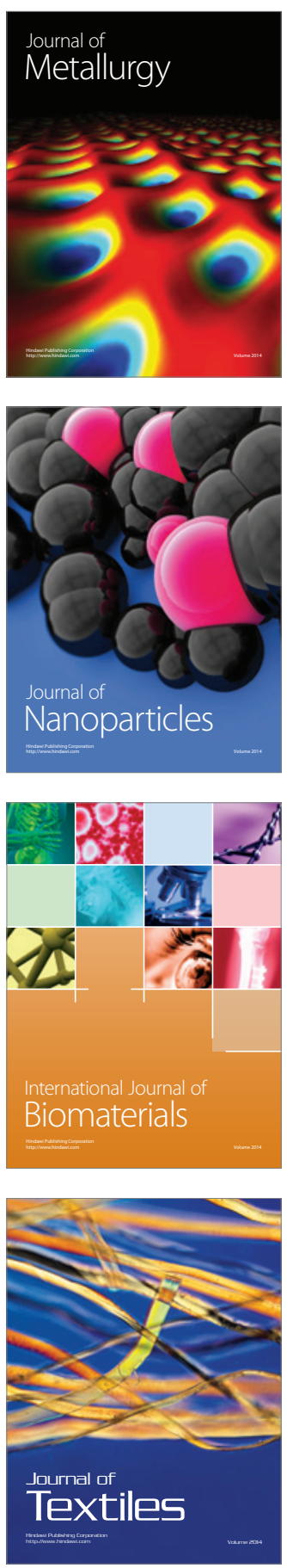